\title{
-NOTES-
}

\section{ON SOLVING SECOND ORDER NONLINEAR DIFFERENTIAL EQUATIONS*}

\author{
By CHONG-HUNG ZEE† (Curtiss-Wright Corporation)
}

\begin{abstract}
A direct power series substitution technique is proposed to solve second order nonlinear differential equations. The errors in truncated power series are estimated to determine the significance of the result.

1. Introduction. The method of continuous analytic continuation has been described as a very powerful computing device in solving nonlinear differential equations [1]. Basically it is a finite-difference method of integrating differential equations based on the assumption that, locally, the desired function may be approximated by Taylor's series in the independent variable. In obtaining this series, many derivatives must be computed and this becomes tedious as the number of terms increases. There is therefore a practical limit to the number of terms, and the result will be in a form of a truncated series. This truncated series is employed over a range that is limited by accuracy requirements. At end of the interval a new series development is started, and so on. The solution, thus obtained, will naturally involve errors. Since there is always a small error in the initial conditions for each iteration, a precise value of the error cannot usually be obtained. To determine the significance of the result, it is suggested that the accuracy of the initial conditions for each iteration be examined. This paper proposes a method of solving nonlinear differential equations more directly and obtaining better estimates of errors.
\end{abstract}

2. Direct power series substitution technique. Assume that the function $y(x)$ is a solution of the second order nonlinear differential equation

$$
y^{\prime \prime}=f\left(x, y, y^{\prime}\right)
$$

with the initial conditions $y\left(x_{0}\right)=y_{0}, y^{\prime}\left(x_{0}\right)=y_{0}^{\prime}$, where the prime denotes differentiation with respect to $x$. Assume further that $y(x)$ is single-valued and analytic throughout some interval $\left(x_{0}, x_{1}\right)$.

Equation (1) can be solved by Picard's method [2] which is essentially an iterative process of developing a series solution. Thus the solution of Eq. (1) may have the form

$$
y(x)=a_{0}+a_{1} \Delta x+a_{2}(\Delta x)^{2}+a_{3}(\Delta x)^{3}+a_{4}(\Delta x)^{4}+\cdots,
$$

where $\Delta x=x-x_{0}$ and $a_{0}, a_{1}, \cdots$ are constants to be determined. If $\Delta x$ is smaller than the radius of convergence for Picard's method, the series in (2) is convergent.

By differentiating with respect to $x$, the following relations are obtained:

$$
\begin{gathered}
y^{\prime}(x)=a_{1}+2 a_{2} \Delta x+3 a_{3}(\Delta x)^{2}+4 a_{4}(\Delta x)^{3}+\cdots, \\
y^{\prime \prime}(x)=2 a_{2}+6 a_{3} \Delta x+12 a_{4}(\Delta x)^{2}+20 a_{5}(\Delta x)^{3}+\cdots .
\end{gathered}
$$

Substituting (2), (3), and (4) into (1) and comparing equal powers of $x$, all coefficients $a_{k}$ are readily determined; in particular, $a_{0}=y_{0}$ and $a_{1}=y_{0}^{\prime}$.

This direct power series technique is particularly powerful in the solution of a system of second order nonlinear differential equations such as

$$
y^{\prime \prime}(x)=f_{1}\left(x, y, y^{\prime}, z, z^{\prime}\right),
$$

*Received April 1, 1963; revised manuscript received Aug. 5, 1963.

$\dagger$ Now Grumman Aircraft Engineering Corporation, Bethpage, N. Y. 


$$
z^{\prime \prime}(x)=f_{2}\left(x, y, y^{\prime}, z, z^{\prime}\right) \text {. }
$$

Again the solutions, $y(x)$ and $z(x)$, are assumed to be single-valued and analytic throughout the interval $\left(x_{0}, x_{1}\right)$ with the initial conditions $y\left(x_{0}\right)=y_{0}, y^{\prime}\left(x_{0}\right)=y_{0}^{\prime}, z\left(x_{0}\right)=z_{0}$ and $z^{\prime}\left(x_{0}\right)=z_{0}^{\prime}$. When a power series of the form in Eq. (2) for $y(x)$ and a similar one with coefficients $b_{0}, b_{1}, \cdots$ for $z(x)$ are substituted into the system of (5), (6), all coefficients are readily obtained.

It is obvious that this technique is easily extended to a larger system of second order nonlinear equations.

3. Accuracy. As is expected in developing any power series, there is a practical limit to the number of terms because of the tedious work involved. Thus series such as in Eqs. (2), (3) and (4) must be in truncated forms, which will give results with truncation errors. An estimate of these errors should constitute part of the solution.

Considering the following series.

$$
\begin{gathered}
y(x)=a_{0}+a_{1} \Delta x+a_{2}(\Delta x)^{2}+a_{3}(\Delta x)^{3}+\cdots+a_{n}(\Delta x)^{n}+R_{n 0}=y_{T}(x)+R_{n 0}, \\
y^{\prime}(x)=a_{1}+2 a_{2} \Delta x+3 a_{3}(\Delta x)^{2}+\cdots+n a_{n}(\Delta x)^{n-1}+R_{n 1}=y_{T}^{\prime}(x)+R_{n 1}, \\
y^{\prime \prime}(x)=2 a_{2}+6 a_{3} \Delta x+12 a_{4}(\Delta x)^{2}+\cdots \\
+n(n-1) a_{n}(\Delta x)^{n-2}+R_{n 2}=y_{T}^{\prime \prime}(x)+R_{n 2},
\end{gathered}
$$

where $\Delta x=x-x_{0}$ and the subscript $T$ represents the truncated series value. If these series are regarded as Taylor's series, then the remainders, in the Lagrangian form are

$$
\begin{aligned}
R_{n 0} & =\frac{1}{(n+1) !}(\Delta x)^{n+1} y^{(n+1)}\left(\xi_{0}\right), & & x_{0}<\xi_{0}<x, \\
R_{n 1} & =\frac{1}{n !}(\Delta x)^{n} y^{(n+1)}\left(\xi_{1}\right), & & x_{0}<\xi_{1}<x, \\
R_{n 2} & =\frac{1}{(n-1) !}(\Delta x)^{n-1} y^{(n+1)}\left(\xi_{2}\right), & & x_{0}<\xi_{2}<x,
\end{aligned}
$$

while all $\xi$ 's have different values and depend to some extent on the magnitude of $\Delta x$. If $\Delta x$ is small, the following approximation can be made $\$$ :

$$
y^{(n+1)}\left(\xi_{0}\right)=y^{(n+1)}\left(\xi_{1}\right)=y^{(n+1)}\left(\xi_{2}\right)=y^{(n+1)}\left(\xi_{m}\right), \quad x_{0}<\xi_{m}<x,
$$

where $y^{(n+1)}\left(\xi_{m}\right)$ is the mean value of $y^{(n+1)}(x)$ in the interval $\Delta x$. Hence

$$
R_{n 0}=\frac{\Delta x}{n+1} R_{n 1}=\frac{(\Delta x)^{2}}{n(n+1)} R_{n 2} .
$$

Substitution of Eqs. (7), (8) and (9) into Eq. (1) and using Eq. (10) yields

$$
y_{T}^{\prime \prime}(x)+\frac{n(n+1)}{(\Delta x)^{2}} R_{n 0}=f\left(x, y_{T}+R_{n 0}, y_{T}^{\prime}+\frac{n+1}{\Delta x} R_{n 0}\right)
$$

which is an equation with $R_{n 0}$ as the only unknown if $\Delta x$ is known. In solving Eq. (11) for $R_{n 0}$ the terms containing $R_{n 0}^{2}$ or its higher powers may usually be omitted; thus $R_{n 0}$ is easily obtained.

In solving a system of second order nonlinear differential equations such as Eqs. (5) and (6), two sets of $R_{n}$ 's will be employed. Each set has the relationship of Eq. (10) and two equations corresponding to Eq. (11) are obtained for solving for the two remainders. Again in solving this set of equations the terms containing $R_{n 0}^{2}$ or higher powers and products of such terms may be omitted. Thus, a set of linear equations

†This approximation has been adopted in estimating the errors in numerical analyses such as Chevilliet's approximation for errors in Simpson's rule [3] and others [4]. 
will finally have to be solved. The same technique can also be employed and extended to a larger system of second order nonlinear differential equations.

It should be pointed out that the primary purpose of estimating errors is to determine the extent to which a given result is significant and not to render it more accurate. When the significance of a result and its desired accuracy are known, the value of $\Delta x$ may be employed over as large a range as possible; thus fewer steps are required for the integration of differential equations over the entire range by the method of continuous analytic continuation.

4. Discussion. After familiarizing oneself with the direct power series substitution technique and better estimates of errors, one finds that only simple algebraic work is involved in solving the nonlinear differential equations. In contrast to this, Picard's method is of limited practical usefulness because of the difficulties involved in integration; furthermore, there is no way to indicate the accuracy of the result. While the classical Taylor's series method has wider application than Picard's method, yet the requirement of higher order derivatives may sometimes lead to difficulties because of the tedious work involved.

The accuracy of a Taylor's series is usually expressed by the magnitude of the remainder of the series, i.e.

$$
\left|R_{n 0}\right|<\operatorname{Max} .\left|\frac{(\Delta x)^{n+1}}{(n+1) !} f^{(n+1)}(\xi)\right|, \quad x_{0} \leq \xi \leq x,
$$

and the corresponding remainder obtained by the present analysis is

$$
R_{n 0} \simeq \frac{(\Delta x)^{n+1}}{(n+1) !} f^{(n+1)}\left(\xi_{m}\right), \quad x_{0}<\xi_{m}<x
$$

Since, by definition

$$
\left|f^{(n+1)}\left(\xi_{m}\right)\right|<\operatorname{Max} .\left|f^{(n+1)}(\xi)\right|, \quad x_{0} \leq \xi \leq x,
$$

the present analysis offers a better estimation of errors than the Taylor's series.

With few exceptions, numerical methods usually do not distinguish between equations which are linear and those which are not; thus the proposed method can also be applied to the linear differential equations.

This direct power series substitution technique with error estimate has been successfully applied to solving the second order nonlinear differential equations of the powered-flight trajectories of rockets $[5,6,7]$. These three papers may serve as examples for the method proposed in this paper.

\section{REFERENCES}

1. Harold T. Davis, Introduction to nonlinear differential and integral equations, Dover Publications, Inc., New York, 1962, pp. 247-266

2. R. A. Buckingham, Numerical methods, Sir Isaac Pitman \& Sons, Ltd., London, 1957, pp. 180-184

3. Ibid, p. 88

4. J. B. Scarborough, Numerical mathematical analysis, The Johns Hopkins Press, Baltimore, 1958, 4th Edition, pp. 174-176

5. C. H. Zee, Rocket in drag-free vertical powered flight under constant thrust, to appear in J. Astronautical Sci.

6. C. H. Zee, Powered fight trajectories of rockets under oriented constant thrust, AIAA J. 1 (1963) 602-606

7. C. H. Zee, Spatial powered flight trajectories of space vehicles under oriented constant thrust, Astronautica Acta, 9 (1963) 107-114 